\title{
INTRAOSSEOUS HAEMANGIOMAS ON HYBRID IMAGING: A PICTORIAL REVIEW
}

\author{
Mairah Razi ${ }^{1}$, Humayun Bashir ${ }^{1}$, Saima Riaz ${ }^{1}$, Zia S. Faruqui ${ }^{2}$ \\ ${ }^{1}$ Department of Nuclear Medicine, Shaukat Khanum Memorial Cancer Hospital and Research Centre, Lahore, Pakistan, \\ ${ }^{2}$ Department of Radiology, Shaukat Khanum Memorial Cancer Hospital and Research Centre, Lahore, Pakistan \\ Received: 26 February 2016 / Accepted: 2 August 2016
}

\begin{abstract}
Osseous haemangiomas are benign skeletal tumours, usually identified as incidental findings on different imaging modalities. Bone scan is the most frequent radionuclide procedure performed as metastatic workup in patients with various malignancies. Not every hotspot on a staging bone scan is malignant. Haemangiomas with variable degree of radiotracer uptake on technetium- $99 \mathrm{~m}$ (Tc-99m) bone scintigraphy may be falsely labelled as metastases in background of known malignancy. The addition of single-photon emission computed tomography-computed tomography (SPECT-CT) enhances the specificity of bone scan which allows accurate detection and anatomical localisation of scintigraphic findings. We present a case series as pictorial review of osseous haemangiomas identified on Tc-99m methylene diphosphonate SPECT-CT at our department.
\end{abstract}

Key words: Haemangioma, methylene diphosphonate, bone scan, single-photon emission computed tomographycomputed tomography

\section{Introduction}

Haemangioma is a slowly growing non-malignant bone lesion. It is characterised by vascular spaces lined with endothelial cells usually located in the medullary cavity. It constitutes approximately $1 \%$ of all primary bone tumours. Spine and skull are the most common sites for haemangioma, representing $75 \%$ of the lesions. The remaining sites may involve tibia, femur and humerus. The sternum is a highly uncommon location. ${ }^{[1]}$ Mostly, these are multiple in up to one-third of the cases and the peak incidence is in the fourth or fifth decade. There is hamartomatous proliferation of vascular tissue within the endothelium which may also contain fat, smooth muscle, fibrous tissue and thrombus. Histological subtypes include intraosseous cavernous or capillary haemangioma, however, may also occur as arteriovenous and venous subtypes. Vertebral haemangiomas are generally capillary type and may cause neurological symptoms if they extend into the epidural canal. Cavernous haemangiomas are common in the skull. ${ }^{[2]}$ Haemangiomas are largely asymptomatic, usually found incidentally on radiography or autopsy.

Correspondence: Dr. Mairah Razi, Department of Nuclear Medicine, Shaukat Khanum Memorial Cancer Hospital and Research Centre, Lahore, Pakistan.

Email: m_sdr@yahoo.com
Haemangiomas mostly appear as round or oval radiolucent lesions on radiographs. A characteristic finding on X-ray will be fine spiculae emanating from its centrum giving a corduroy, spoked wheel or honeycomb appearance. ${ }^{[1]} \mathrm{On}$ axial computed tomography (CT) scan, vertebral body lesions have a typical spotted 'polka dot' pattern due to thickened trabeculae appearing as small punctate areas of sclerosis in cross-section. Calvarial haemangiomas are usually lytic and trabecular thickening resemble as radiating, web-like or spoked wheel pattern. In long bones, the metaphyseal or epiphyseal lesions appear lytic that gives a spiculated array like an 'Irish lace.' Trabecular pattern of vascular malformations of the bone usually displays a high signal intensity on magnetic resonance imaging (MRI). However, these features may vary; largely depend on the proportion of fat and vascularity of the lesions. T1-weighted MRI scans diverge from low to high intensity depending on the content of adipose tissue present. T2-weighted MRI scans display lesions with high intensity due to the vascularity. Bone scintigraphy is usually normal but may show increased or decreased uptake in haemangiomas. ${ }^{[3]}$

\section{Image Acquisition}

Planar bone scintigraphy acquired after $20 \mathrm{mCi}$ of technetium-99m (Tc-99m) methylene diphosphonate (MDP). 


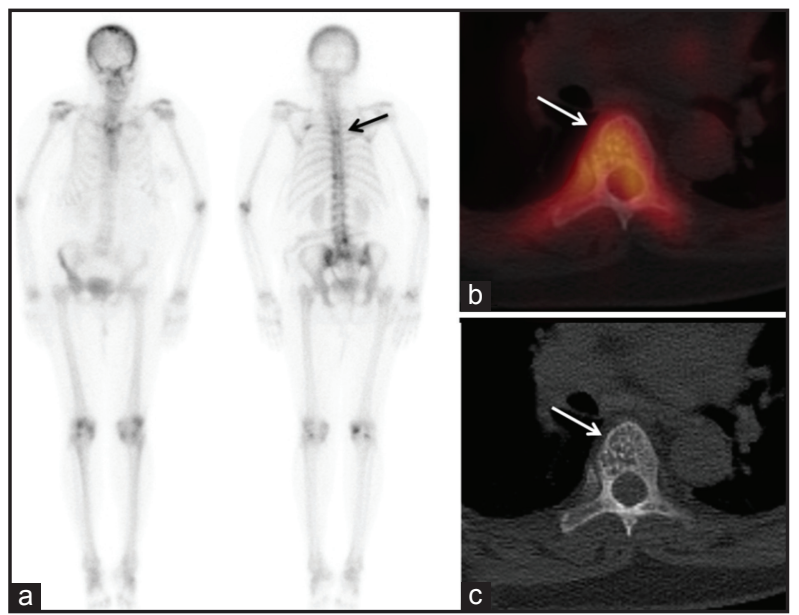

Figure 1: A 59-year-old female patient with breast carcinoma. (a) Whole body technetium-99m methylene diphosphonate static images showing increased tracer uptake at thoracic (D6) vertebra (arrows), (b) Fused axial single-photon emission computed tomography-computed tomography $(\mathrm{CT})$ demonstrate increased uptake in D6 vertebra and (c) axial CT images reveal typical 'polka dot' appearance of haemangioma at D6 vertebra (arrows)

Standard single-photon emission computed tomography-CT (SPECT-CT) acquisition parameters were used.

SPECT images acquired using low-energy parallel-hole collimators with large field-of-view gamma detectors (range, $40 \mathrm{~cm}$ ), $180^{\circ}$ arc, $6^{\circ}$ view angle, zoom of 1.0 and $30 \mathrm{~s} / \mathrm{stop}$. Images were acquired with a $128 \times 128$ matrix and then reconstructed using a three-dimensional orderedsubset expectation maximisation iterative technique (eight subsets and four iterations). CT images acquired using single-detector step-and-shoot technique, 10-mm slice interval, current of $2.5 \mathrm{~mA}$, voltage of $140 \mathrm{kV}$, $256 \times 256$ matrix and a Gaussian filter. Total imaging time for SPECT-CT was approximately 25-30 min. [Figures 1-6] represent cases of haemangioma identified with SPECT-CT.

\section{Discussion}

Skeletal scintigraphy has the advantage of entire skeletal visualisation in oncological patients who are at high risk for osseous metastasis. ${ }^{99 \mathrm{~m}} \mathrm{Tc}-\mathrm{MDP}$ is the most commonly available tracer for skeletal imaging. Despite the high sensitivity, not every hotspot on bone scan is malignant.

Variable causes of increased tracer uptake mimicking metastatic disease impair the specificity of the bone scintigraphy. Solitary finding on bone scan often requires further radiologic correlation with $\mathrm{CT}$ to improve the limited specificity of bone scan. ${ }^{[4]}$ This limitation of planar scintigraphy has been overcome by the introduction of hybrid SPECT/spiral CT since 2001. ${ }^{[5]}$

Osseous haemangiomas are usually asymptomatic and the diagnosis is incidental most of the time. Rarely, they appear symptomatic constituting approximately $1 \%$ of all cases. ${ }^{[6]}$

Appearance of haemangioma on bone scan has been well documented in literature. Variable degree of radiotracer uptake is seen in haemangiomas; increased, decreased or equal to adjacent bone. Vertebral haemangiomas which are smaller than $3 \mathrm{~cm}$ in size generally show normal uptake of a radiotracer. However, larger lesions may demonstrate either increased or decreased uptake. ${ }^{[7]}$

They may be erroneously labelled as metastases on bone scan in background of primary malignancy which have predilection for skeletal (lytic) metastases. In addition to post-external radiotherapy, haemangioma is also one of the causes of 'cold' vertebrae on skeletal scintigraphy. ${ }^{[8]}$

Han et al. evaluated case series of 15 vertebral haemangiomas in 10 patients on bone scan. Planar images showed normal findings throughout the skeleton with the exception of only one. SPECT images also displayed normal findings in 11 vertebral haemangiomas which were smaller than $3 \mathrm{~cm}$ in diameter. However, three of four vertebral haemangiomas were $3 \mathrm{~cm}$ or larger and demonstrated variably increased or decreased uptake on SPECT images. ${ }^{[9]}$

The role of SPECT-CT has been reported in a 20-year-old woman with upper back pain by Bhoil et al. Planar bone scintigraphy showed focal uptake in the seventh thoracic vertebra. SPECT-CT showed uptake in the vertebral body and transverse process at D7 with CT findings typical of haemangioma. Furthermore, MRI of the thoracic spine confirmed the findings of haemangioma in the same vertebra. $^{[6]}$

A case of increased tracer uptake in haemangioma has also been reported in a patient of renal cell carcinoma who underwent bone scintigraphy to assess for skeletal 


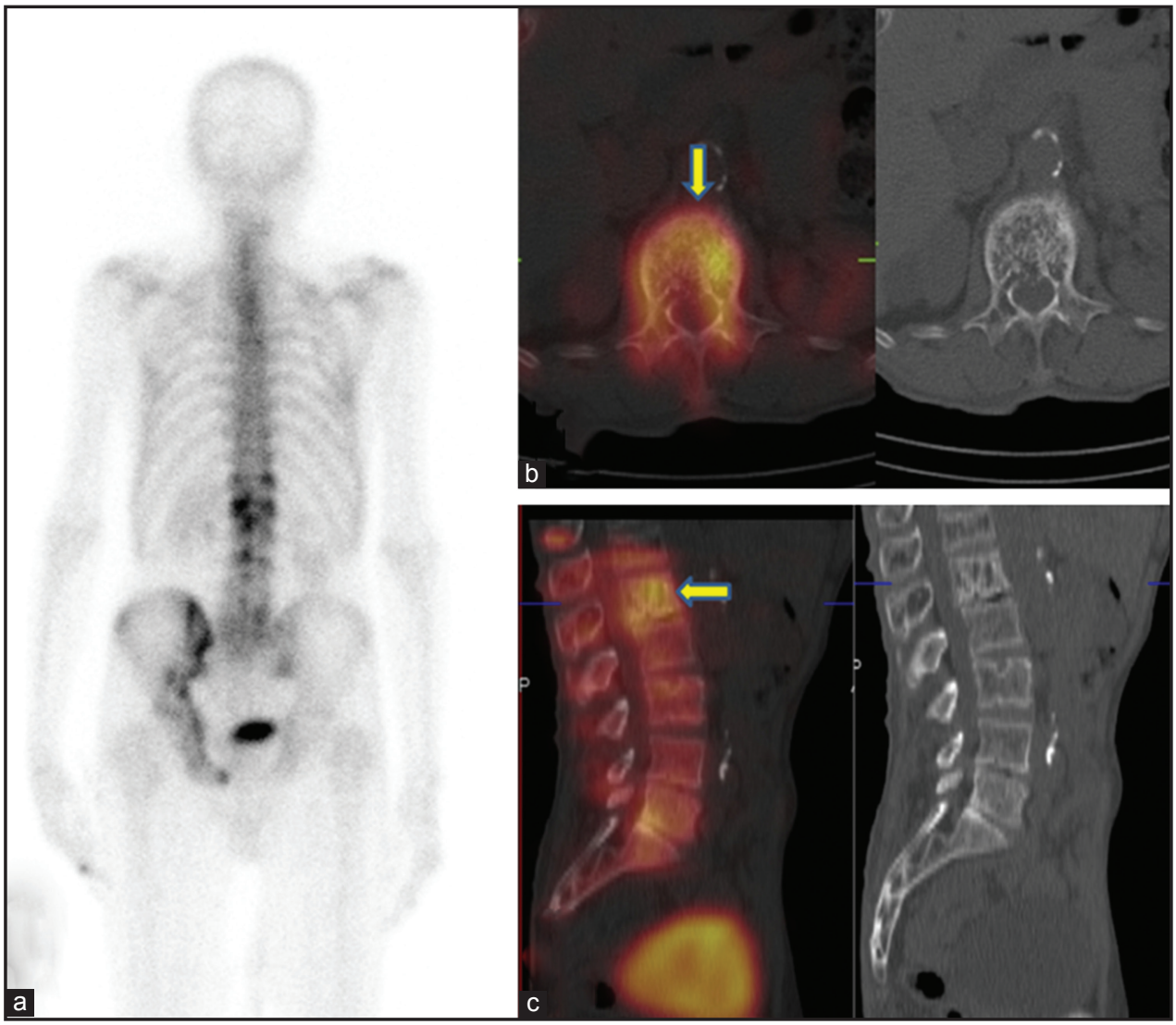

Figure 2: An 81-year-old male patient with temporal glioma. (a) Planar images show variable increased tracer uptake in the thoracolumbar vertebrae, (b) axial and (c) sagittal fused and computed tomography (CT) images of single-photon emission computed tomography- CT bone scan demonstrate haemangioma of L1 vertebra with increased radiotracer uptake. Degenerative changes are noticed at multilevel in the spine and pelvic bones
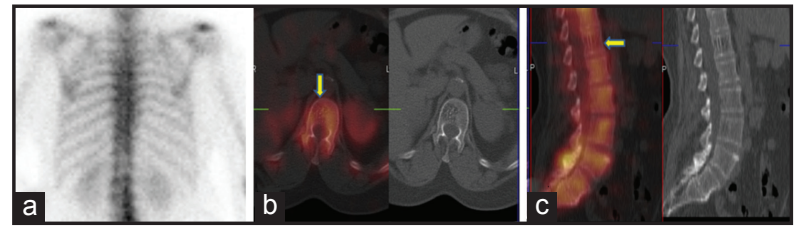

Figure 3: A 58-year-old female patient with breast carcinoma. (a) Planar spot view of the posterior chest shows irregular uptake in the thoracolumbar vertebrae with an area of reduced uptake at D12, (b) axial and (c) sagittal fused and CT images of single-photon emission computed tomography- CT bone scan revealed haemangioma in the D-12 vertebra with decreased radiotracer uptake

metastases. SPECT-CT localised the MDP uptake in D8 vertebra with vertical trabecular thickening typical of haemangioma ruling out metastasis. Furthermore, on review of thoracolumbar spine on $\mathrm{CT}$, similar lesions were identified in D10 and L3 without any significant uptake. ${ }^{[10]}$

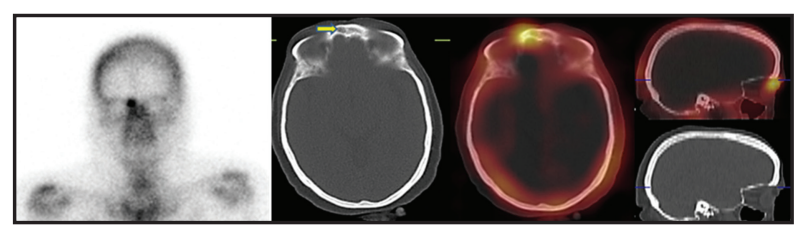

Figure 4: A 64-year-old female patient with breast carcinoma. Planar anterior skull view shows focal increased tracer uptake in the right frontonasal region. Axial/sagittal fused and CT images of single-photon emission computed tomography- CT bone scan demonstrate haemangioma in the frontal bone with increased radiotracer uptake

The reason for increased tracer uptake is haemangiomas not very clear. Calcification in old haemangiomas or sclerotic changes in the involved bone could be considered as few possibilities. In view of a proven malignancy, these features always arouse suspicion of metastatic involvement and hybrid imaging helps in timely characterisation. 


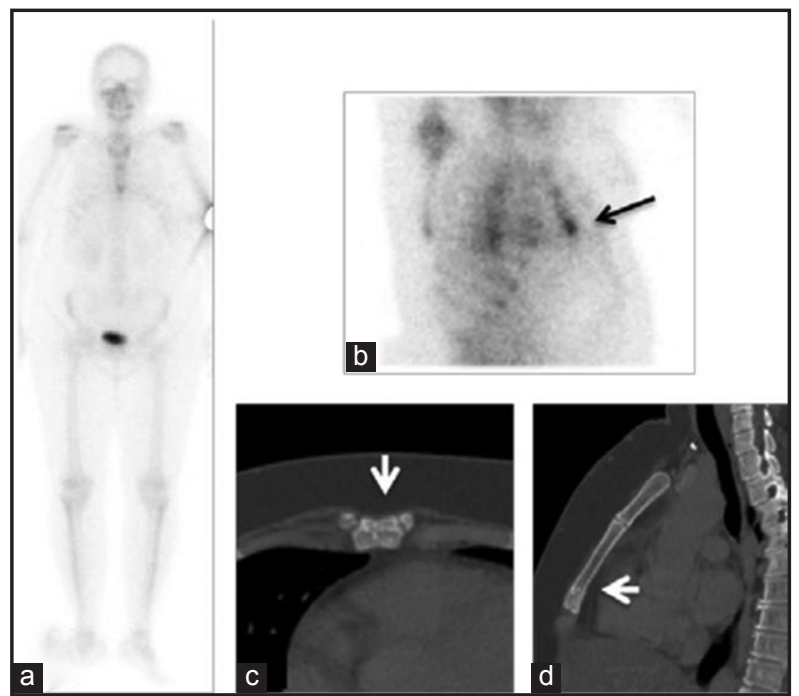

Figure 5: A 43-year-old patient with follicular carcinoma of thyroid. (a) Planar whole body and (b) right anterior oblique views show mild tracer uptake in the tip of the sternum. (c) axial and (d) sagittal views of the conventional computed tomography revealed trabecular thickening at the tip of the sternum - a rare site for haemangioma

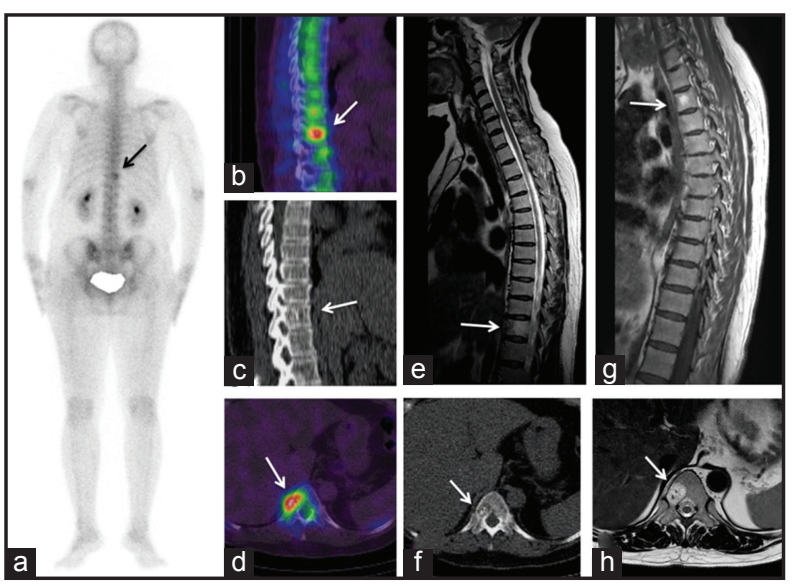

Figure 6: A 55-year-female patient with the left breast carcinoma. (a) Planar images of bone scan showing increased tracer uptake in D11 vertebra. Sagittal and axial fused single-photon emission computed tomography-computed tomography (CT) and CT images (b-e) show typical polka dot appearance at D11 hemivertebra with increased tracer uptake. (f and g) T2-weighted sagittal magnetic resonance imaging (MRI) of the thoracic spine shows multifocal haemangioma in this patient with hyperintense signal in D2 and D11 vertebrae and (h) axial T2-weighted MRI

\section{Conclusion}

Intraosseous haemangiomas show variable Tc-99m MDP avidity. When combined SPECT-CT is used, the benefit of precise anatomic localisation of the Tc-99m MDP uptake and the corresponding CT appearance help characterise the nature of these benign lesions.

\section{Conflict of Interest}

The authors declare that they have no conflict of interest.

\section{References}

1. Franquet T, Giménez A, Alegret X, et al. Imaging findings of sternal abnormalities. Eur Radiol 1997;7:492-7.

2. Raphael J, Hephzibah J, Mani S, et al. Abnormal appearance of spinal hemangioma mimicking metastasis in bone scintigraphy and SPECT-CT: Case report. J Nucl Med Radiat Ther 2013;S6:16-8.

3. Murphey MD, Fairbairn KJ, Parman LM, et al. Musculoskeletal angiomatous lesions: Radiologicpathologic correlation. Radiographics 1995;15:893-917.

4. Even-Sapir E. Imaging of malignant bone involvement by morphologic, scintigraphic, and hybrid modalities. J Nucl Med 2005;46:1356-67.

5. Buck AK, Nekolla S, Ziegler S, et al. SPECT-CT. J Nucl Med 2008;49:1305-19.

6. Bhoil A, Mittal BR, Harisankar CN, et al. Methylene diphosphonate uptake in a case of vertebral haemangiomas: Demonstration with hybrid SPECT/CT. Clin Nucl Med 2011;36:e65-6.

7. Moran DE, O'Neill AC, Heffernan EJ, et al. Not everything that is hot on a staging bone scan is malignant: A pictorial review of benign causes of increased isotope uptake. Can Assoc Radiol J 2012;63:280-8.

8. Domínguez M, Rayo J, Serrano J, et al. Vertebral hemangioma: "Cold" vertebrae on bone scintigraphy and fluordeoxy-glucose positron emission tomographycomputed tomography. Indian J Nucl Med 2011;26:49-51.

9. Han BK, Ryu JS, Moon DH, et al. Bone SPECT imaging of vertebral haemangiomas correlation with MR imaging and symptoms. Clin Nucl Med 1995;20:916-21.

10. Manohar K, Parghane RV, Kumari S, et al. Multiple vertebral haemangiomas mimicking metastases in a patient with renal carcinoma: Importance of hybrid imaging with SPECT/CT and FDG PET/CT. J Postgrad Med Edu Res 2012;46:196-7. 